\title{
Investigation and Prevention of Intestinal Parasitic Infection in Children in Kindergarten in Baoshan City
}

\author{
Yongmei Zhang \\ Baoshan college of traditional Chinese medicine in baoshan city, Baoshan, Yunnan, 678000
}

Keywords: Intestinal Parasitic Infection, Kindergarten, Investigation and Prevention

\begin{abstract}
To understand the infection of intestinal parasites in children in Baoshan area, especially the prevalence of opportunistic protozoa and zoonotic parasites, from October 2007 to May 2008, 8 villages and towns in Baoshan area were surveyed. Using Lugol's iodine staining, saturated sucrose solution floating, modified acid-fast staining and molecular biology method, 1949 children under 15 years of age were surveyed in 23 investigation points of Linzhou area. The total infective rate of parasite was 1.33\% (26/1949). Six kinds of intestinal parasites were found, of which three were parasitic and worm, the infection rates were $0.72 \%$ and $0.62 \%$ respectively. The infection rates of males and females were $1.27 \%(14 / 1103)$ and $1.42 \%(12 / 846)$ respectively, with no significant difference $(\mathrm{P}>0.05)$. The infection rates of children in Yaocun, Chengjiao, Chaandian, Linqi, Wulong, Dongyao, Hengshui and Jinsang were $1.76 \%, 1.42 \%$ and $0.83 \%$ respectively. $71 \%, 0.00 \%$, $0.74 \%, 1.45 \%, 0.75 \%$ and $1.33 \%$, respectively. There was no significant difference in the infection rates among towns $(\mathrm{P}>0.05)$. For the first time, to find out the prevalence of children infected with Cryptosporidium in Baoshan area, the infection rate was $0.15 \%$. One isolate of Carnosoma was molecularly identified, and the sequence analysis showed that Atoxoplasma sp. (AY331571) was the closest, homology reached $99.4 \%$, indicating that the intestinal parasitic infection may be caused by bird fecal contamination. The prevalence of intestinal parasites in Baoshan area is low, but health education should be further popularized, health knowledge should be popularized, self-care awareness and self-care ability should be improved, and the occurrence and prevalence of parasitic diseases should be prevented.
\end{abstract}

\section{Introduction}

Human intestinal parasites is a common disease, frequently-occurring disease, seriously affecting human health, especially the incidence of children is higher, the level of parasitic infection is an important indicator of a region's health level. Parasitic opportunistic pathogens and zoonotic parasites in the gut seriously threaten human health and public health, especially children with immunocompromised diseases, those who are infirm and infirm, those with immunocompromised infections are more susceptible to infection and cause morbidity and mortality, More and more researchers are paying great attention and attention. Data show that children in Henan Province is still at high risk of parasitic infection, the infection rate is relatively high. Linzhou is Hongqiqu's hometown, located in the eastern foot of the Taihang Mountains, Shanxi, Hebei and Henan provinces at the junction of special geographical environment, lifestyle and eating style unique style. In order to understand the infectious status of intestinal parasites in children in Baoshan area and to grasp the epidemic situation of opportunistic pathogenic protozoa and zoonotic parasites, from October 2007 to May 2008, Parasitic infections were investigated

\section{Materials and Methods}

From October 2007 to May 2008, according to the different geographical features and economic level, 23 out of the 16 townships in Linzhou were surveyed, and fresh faeces of children under 15 years old were collected for about $20 \sim 50$ g, respectively, into a clean plastic bag, number and register for more information. A total of 1949 people were surveyed, of whom 56 were 0-2 years old, 1622 were 3-6 years old, 219 were 7-10 years old and 52 were 11-15 years old. The collected fecal 
samples were stored in a refrigerator at $4{ }^{\circ} \mathrm{C}$ and tested within $24 \mathrm{~h}$.

The stool samples collected were directly observed under iodine light, saturated sucrose solution floating method and modified acid-fast staining method.

The isolates of Oocysts of Oocysts were picked out with a self-made plastic capillary tube under an inverted microscope. A total of 100 oocysts were placed in a $1.5 \mathrm{ml}$ Eppendorf tube. The DNA extraction was performed using the Mag Extractor-Genome kit manufactured by TOYOBO Co., Ltd., Japan. The extraction procedure was the same as the previous method. Five NCBI isolates were sequenced on the NCBI using the BLAST tool: Isospora suis (ISU97523), I. ohioensis (AF029303), I. robini (AF080612), I felis (L76471), I. beill (DQ060658), aligned with OMIGA (version 2.0) with a threshold of $100 \%$ to generate a consistent sequence. Then use Primer 5.0 to design a set of nested PCR primers. The primers were: upstream 5'-TGTTTATTAGATACAGAACCAACCCAC-3', downstream 5'-CTTTCTATGTCTGGACCTGGTGAG-3', and the amplified fragment was about $1088 \mathrm{bp}$; inner primer: upstream 5'-TAACCGAACGGATCGCGTTAT-3', downstream 5'-GCCGAAATCCTATCTTATTATTCC-3' the amplified fragment is about 530bp. The primers were synthesized by Shanghai Bio Co., Ltd., and the PCR amplification enzyme was KOD-Plus amplification enzyme produced by Japan TOYOBO Corporation. The annealing temperatures used for PCR and internal primers were $52^{\circ} \mathrm{C}$ and $55^{\circ} \mathrm{C}$, respectively. The PCR product was sequenced by Po Bio (Dalian) Engineering Co., Ltd.

The survey found a total of 26 cases of intestinal parasites, the total infection rate was $1.33 \%$ (26/1949). Six human intestinal parasites were found, Giardia, Cryptosporidium, pinworm, roundworm, hookworm and isolates of coccidia. Among them, Giardia infection rate was 0.41\% (8/1949), cryptosporidium infection rate was $0.15 \%$ (3/1949), pinworm infection rate was $0.56 \%$ $(11 / 1949)$ the rate of hookworm infection was $0.05 \%(1 / 1949)$ and the rate of isolates of carnosphaeria was $0.05 \%$ (1/1949). No other parasites and mixed infection. The infection rate was highest at $7-10$ years old, $1.83 \%$. The infection rate was $1.36 \%$ at $3-6$ years old. No parasitic infection was found in 0-2 years old and 11-15 years old. There was no significant difference in infection among children of different ages.

The prevalence rates of children in Yao villages, Chengjiao townships, Chadian township, Linqi town, Wulong town, Dongyao town, Hengshui town and Jinsang district were $1.76 \%, 1.42 \%$ and $0.83 \%$ respectively, $2.71 \%, 0.00 \%, 0.74 \%, 1.45 \%, 0.75 \%$ and $1.33 \%$ respectively. There was no significant difference among towns ( $\mathrm{P}>0.05)$. To Lin Chi highest prevalence of children in the town of Wulong children found no intestinal parasitic infection. Giardia and Cryptosporidium are mainly distributed in Yao Village, Chengjiao Township and Linqi Town.

Fifty oocysts were assayed with an average size of $22.13 \times 19.67 \mu \mathrm{m}$ and a sporangia size of $14.75 \times 9.84 \mu \mathrm{m}$. The amplified PCR product was then sequenced to obtain a $539 \mathrm{bp}$ fragment of interest. Through software analysis, a coccidian of Atoxoplasma sp. (AY331571), the number of nucleotide differences was 3 and the homology was $99.4 \%$. But only $85.9 \%$ homology with Isospora belli (DQ060658), a searchable parasitoid in the human body.

\section{Discussions}

Baoshan City in 2002-2004 carried out the second investigation of the status quo of human parasitic diseases, found 29 kinds of intestinal parasites, including 18 kinds of worms, 11 kinds of protozoa, the total intestinal infection rate was $14.96 \%$ Among them, the worm infection rate was $9.80 \%$, protozoal infection rate was $6.61 \%$, and the mixed infection rate was $9.32 \%$ Zhang Hongwei et al conducted a survey of human intestinal parasites in Henan Province in 2003, and detected 17 kinds of intestinal parasites, 14 kinds of worms and 3 kinds of protozoa, with a total infection rate of $9.63 \%$. Among them, worm infection the rate was $7.70 \%$, the protozoal infection rate was $2.15 \%$ and the mixed infection rate was $0.57 \%$. Yuyouyong et al conducted a survey of intestinal parasitic infections in Anyang City in 2002 and found that there were 6 kinds of parasites: roundworm, whipworm, hookworm, pinworm, giardia and gondii cyst, the total parasites infection rate was 4.11\%, no mixed infection was found. The investigation of children in Linzhou district found 6 
kinds of intestinal parasites: Giardia, Cryptosporidium, coccidia, pinworm, roundworm and hookworm, the total infection rate was $1.33 \%$, the protozoal infection rate was $0.62 \%$. The worm infection rate was $0.72 \%$. Child worm infection was mild and no mixed infection was found. The survey's infection rate and infection intensity were significantly lower than those of Xu Haili et al. And Zhang Hongwei et al., Which were lower than those of Yu Yongyong et al.

Lai et al survey of children, 0 to 4 -year-old infection was $25.86 \%, 5$ to 10 -year-old infection was $66.23 \%, 11 \sim 15$-year-old infection was $7.91 \%$. The survey of 3 to 6 years of age as the main infection age, the proportion of $84.62 \%, 7$ to 10 years of age accounted for $15.38 \%$ of age, 0 to 2 years old and children aged 11 to 15 found no parasites Insect infection, consistent with the data. 0 to 2-year-old children are strongly protected by their parents and have a lower risk of infection; 11 to 15-year-olds already know how to manage their own health and hygiene and are less likely to become infected; 3 to 10-year-olds have poor awareness of disease prevention and behavioral selfExposure to infection is more likely. Li Hui and other reports in 1990, Anyang City, male and female intestinal parasites infection rate differences were significant in 2002 survey of men and women infection rate was no significant difference; Zhang Hongwei et al The survey showed that different sexes intestinal parasites infection difference Statistical significance; Shandong Province conducted in 2002-2003 survey of the status of important parasitic diseases showed no significant difference between men and women infection rate. In this survey, parasitic infections among men and women were different. The prevalence of intestinal parasites in females was slightly higher than that in males ( $\mathrm{P}>0.05)$. The average age of different gender children the infection rates of women aged $3 \sim 6$ years and $6 \sim 10$ years were higher than those of men. The analysis showed that there was no significant difference $(\mathrm{P}>0.05)$. Ascaris, pinworm and whipworm and other soil-borne nematode infections showed significant local and family aggregation, and is closely related to the economic level. The survey found that the worm infection rate was significantly lower, only $0.62 \%$, did not find whipworm infection. Data show that Henan Province, the rate of intestinal parasites showed a significant downward trend and socio-economic factors are closely related. In recent years, the economic development of Linzhou has been steadily developing. The decline of the parasitic infection rate shows that the improvement of the economic level has played an important role in the prevention and treatment of parasitic diseases.

Giardia and Cryptosporidium are important zoonotic opportunistic pathogens. Giardia is an estimated 200 million people in Asia, Africa and Latin America, accounting for about 500,000 new infections each year. The first national survey of human parasites in 1990 found that Giardia infection rate was $2.52 \%$, Zhang Hongwei and other survey results showed that Giardia in Henan Province, the highest rate of human intestinal parasitic protozoa, 2.04\%. The survey found that Giardia infection rate was $0.41 \%, 3$ to 6 years old as the main age of infection, the highest infection rate of Linqi children, was $1.42 \%$. Cryptosporidium is one of the common pathogens causing diarrhea in humans and mainly infects immunodeficient patients such as AIDS patients and children. According to Xu Longqi and other statistical analyzes in 2000, 20977 diarrhea children in 14 provinces (cities and districts) were investigated in 1998, and 449 cases of cryptosporidiosis were found, with an average infection rate of $2.14 \%$. The survey for the first time found out Baoshan area of children infected with Cryptosporidium infection rate was $0.15 \%$. Since the molecular biology of the isolated Cryptosporidium has not been performed, it is not possible to determine the source of infection and the route of transmission.

\section{Conclusions}

The survey results show that young age is higher than the prevalence of infection, boys than girls infected with high rates of rural students infected than urban students. The prevalence of intestinal parasites is a mirror of socioeconomic status, reflecting environmental hygiene practices and a marker of health and hygiene awareness. These three infection rates are high, may be associated with the younger the age of young people, the weaker self-control ability, the health awareness of the worse; boys than girls range of activities, in the ground and field contact with the earth to play more; rural poor sanitary conditions, lack of health knowledge and Bad hygiene and other related. 
This shows that it is very important that young people and rural areas actively carry out a wide range of comprehensive prevention and control of parasitic diseases, improve their overall quality and ensure their health.

\section{Acknowledgements}

The general subject of baoshan city technology bureau - "the investigation about intestinal parasitic infection situation of children in kindergarten of baoshan city urban area and suburbs ", project number: 2017 kj012

\section{References}

[1] Wang R, Zhang L, Ning C, et al. Multilocus phylogenetic analysis of Cryptosporidium andersoni (Apicomplexa) isolatedfrom a bactrian camel (Camelus bactrianus) in China [J]. Parasitol Res, 2008, 102 (5): 915-920.

[2] Xiao LH, Fayer R. Molecular characterization of species and genotypes of Cryptosporidium and Giardia and assessment of zoonotic transmission [J]. Int J Parasitol, 2008, 38 (11): 1239 -1255.

[3] Yason JA, Rivera WL. Genotyping of Giardia duodenalisisolates among residents of slum area in Manila, Philippines [J]. Parasitol Res, 2007, 101 (3): 681-687.

[4] Li Hui, Lin Ximeng, Huang Qian, and so on. Analysis of intestinal parasitic infection in Baoshan population [J]. Chinese Journal of Parasitic Diseases, 2004, 17 (4): 223-225.

[5] Wang Fang, Hu mountains, Zhu Jingjing, and so on. Epidemiological survey of quail Cryptosporidiosis in Baoshan City [J]. Chinese Veterinary Science, 2008, 38 (1): 77-81. 